\title{
Teaching Objective Evaluation Paradigm Based on "Output" (OBE)
}

\author{
Zhaoling $^{1, *}$, Zhaoqianqian ${ }^{2}$, Liyanni $^{3}$ \\ ${ }^{1,2,3}$ National university of defense and technology, information and communication college \\ *Corresponding author Email id: 524864718@qq.com
}

\begin{abstract}
Talent training quality is the lifeline of higher education, this article on the basis of reference to engineering concept, based on the evaluation of "paradigm", to develop professional level of expectations "learning outcomes", centered on students' learning and development, the results orientation and continuous improvement education idea guidance, practice how "quality consciousness, quality, the quality of the Chinese revolution" internalized as endogenous consciousness and behavior of teachers and students on own initiative, provide a directional course control and innovation ability of our country's enlightenment and reference.
\end{abstract}

Keywords: OBE, "output", objective evaluation

\section{基于 “产出式”（OBE）的教学目标评价范式}

\author{
赵玲 ${ }^{1}$ ，赵倩倩 ${ }^{2}$, 李艳妮 ${ }^{3}$
}

${ }^{123}$ 国防科技大学信息通信学院试验训练基地, 西安, 陕西, 中国 a524864718@qq.com

\section{摘要}

人才培养质量是高等教育的生命线，本文在借鉴工程学概念的基础上，立足于评价 “范式”，制定专 业层面的预期 “学习产出”，以学生学习和发展为中心，在成果导向和持续改进教育理念引导下，践 行了如何将 “质量意识、质量革命、质量中国” 内化为教师和学生的内生意识和行为自觉, 为我国课 程的驾驭与创新能力提供方向性的启示与借鉴。

关键字: $O B E 、 “$ 产出式”、目标评价

\section{1. 前言}

当下, “新科技革命”和“新工业革命”的在全世 界 范围内的竞争态势愈演愈烈, 也使我国高等教育面临 着前所未有的机遇和挑战, 新工科背景下相关专业及 课 程体系的教学目标、授课内容、支撑的毕业能力要 求、达成方式及考核方式等函待重塑和转型, 其核心 要义是要求符合“产出导向 (Outcome-Based Education, 简称 OBE）”的教育理念。

\section{2. 解析成果导向的教学目标评价范式}

1981 年, 美国学者斯派帝 (Spady) 提出了 OBE 的教育理念, 这也是一种以能力需求本位为导向的教 育最早出现在公众视野, 由于这一时期人们更加关注 教育投入回报与实际产出的现实需要, 一度成为西方
国家所追捧的教育理念，对北美及西欧的基础教育改 革均产生深远影响。

\section{1 成果导向与目标产出}

“产出式”（OBE）的教学目标评价范式, 顾名思 义, 即学生产出驱动教育系统运行的教学目标评价范 式, 是学习产出驱动整个课程活动和学生学习产出评 价的教育的结构模式与系统, “清晰地聚焦和组织教育 系统, 使之围绕确保学生获得在未来生活中获得实质 性成功的经验。[1]“范式”（Paradigm）最早由美国学 者 $\mathrm{T}$. 库恩在《科学革命的结构》一书中提出, 指在 流变的、开放的专业领域或学科中寻找理性的、共通 的共同信念, 框架性地为解决问题提供观念和方法的 科学指导, 后多被转译为“范型”、“规范”。

著名的“泰勒原理”科学地解决了课程编制必须回 答的四个基本问题: 确立教学目标、选择学习经验, 
组织学习经验和评价学习结果, 在泰勒(Ralphw.Tyler) 看来, 评价不是客不客观的问题, 而是教育目标是否 实现的问题, 评价是泰勒模式的最后步骤, 但并非最后 进行, 而是在整个规划过程中就已经出现。Acharya 据 此提出了 $\mathrm{OBE}$ 教育模式的四个步骤：定义学习产出 (Difining) 、实现学习产出（Realizing）、评估学习 产出 (Assessing) 和使用学习产出 (Using), 涵盖了计 划、实施、检查、行动等要素, 关注学生的目标达成 度, 以预期学习产出为中心来组织施教, 评估学习产 出, 检测学习结果, 可以完美实现现实意义上的教育 范式转换。

\section{2 以师生“双主体”为中心}

教学评价是以教学目标为依据, 按照科学的标准, 运用一切有效技术手段, 对教学过程及结果进行测量, 并给予价值判断的过程, [2] 主要包括对学生学业成绩 的评价以及对教师教学质量的评价。这就意味着在 OBE 教育系统中, 师生 “双主体” 的授受关系举足轻 重, 教育者必须对受教育对象现有的能力程度及通过 努力后能达到的发展水平有清晰的预判和把握, 并能 够依据受教育对象的知识能力水平程度寻求设计适宜 的教育结构来实现教育活动的预期目标。

根据布鲁姆的掌握学习目标理论, 如若提供足够 的学习时间和适宜的学习机会, 任何人都能实现并达 到对学习目标的掌握程度。OBE 在很多方面吸纳了掌 握学习理论的特征, 如: 使用不同的资源去创设丰富 的教育环境; 提供可选择的弹性时间去实现教学目标; 使用形成性教学评价来反馈学习产出以改善教与学等, 通过测验来了解学生掌握知识和能力的水平, 查漏补 缺, 让每个学生都能获得学业成就感。可以说, 以师 生 “双主体” 的理论架构是支撑 OBE 教学目标评价范 式的重要假设之一。

\section{3 持续改进与能力生成}

成果导向直指我们可以得到什么有形的输出物, 但 需要强调的是, 基于成果导向与目标产出但成果导 向并不意味着只重视结果忽视学习过程。目前, 人们 对课堂评价的研究已经由以关注目标评价为核心思想 的泰勒评价模型和类泰勒评价模型的研究, 转向了以 关注主体生成为核心理念的新课堂评价模型的研究,

“教育结构和课程是手段而非目的, 若它们无法为培 养学生特定能力作出贡献, 它们就要被重建”。

发展性教育评价发端于泰勒的目标评价思想, “学生学到了什么和是否成功远比怎样学习和什么时 候学习重要”, 泰勒模式主张采取参照实验, 而基于 “产出式” 的教学目标评价范式弥补了泰勒模式的不 足, 同时吸收了斯里克温、斯坦豪斯等人的新潮评价 观的优势，基于目标产出反思和改进认知加工的实践, 倡导导向思维意识, 强调个性化评定, 认为只要给学
生适宜的机会及合理的评定, 所有的学生都能达到满 意的学习结果, 根据学生需要重新设置课程目标, 重 构课程知识单元，建立教学效果评价反馈机制。

产出而非教科书或导生式的师生授受为内驱力的 教育系统运作，这显然与传统意义的资金配比倾斜及 内涵 式驱动的教育形成鲜明对比。从这个意义上说, OBE 教育教学目标评价范式可被认为是评价范式领域 的一次革新。

\section{3. $\mathrm{OBE}$ 评价指标的确立及测评方法的选择}

当前, 教学评价正处于范式转换时期, 探寻范式 特别是教学评价的结构与要素、指标与方法, 对解决 人们正面临的许多教学评价新情况、新问题具有重要 的现实意义。

\section{1 评价的分类}

布鲁姆根据评价在教学中的功能, 将评价进行分 类: 诊断性评价, 即前侧, 是在教学初始对学生已有 的知识和能力水平的测试, 其目的是为了有的放矢地 因材施教, 如: 摸底考试; 形成性评价, 又称进展性 测评, 在教育 教学过程中组织实施, 通过正在进行的 师生间教学交往和互动了解教学情况进展, 如: 随堂 小测试; 终结性评价, 是某一阶段或某一课程终结时 对学生学习成绩的综合评定, 或被认为是获取某种资 格的认定依据, 如: 我们最为熟知升学、等级考试。

评估学习产出是 OBE 教育模式中十分重要的环节, 也是牵扯教师精力最多、最复杂的环节。如, 美国成 立的 “全国学习产出评估机构” , 立足于高校建设发 展组织建立全国性的学情数据库系统, 鼓励各高校积 极参与、企业参与论证以及评估中心定期进行学生评 估, 开发了很多学校层面的、用于评估学生学习产出 的工具, 最终结果是形成了这为动态地跟踪高等教育 质量以及 “学生学习增殖” 提供了有效证据和有力的 信息反馈来源。从本质上讲, OBE 教育模式就是围绕 “定义预期学习产出一实现预期学习产出一评估学习 产出” 这条主线而展开, 学生产出评估构成了教育质 量持续改进的闭环。

\section{2 评价的指标}

定义毕业生预期学习产出是首要的关键环节，基 于 “产出式” 的教学目标评价范式必须建立可操作化、 量化和具体的指标体系。

布鲁姆的教育目标分类是目前被学界广泛采用的 教育指南, 也是 $\mathrm{OBE}$ 教学评价的重要指标: 知识、能 力、情感三维目标。在知识领域, 从简单的、具体的 事物形象, 经过头脑认知加工, 全面、综合分析事物, 得到抽象、概括性的认识, 最后能够做出合乎理性的 正确的思维、理解和判断, 这一过程可简要概括为识 
记、领会、应用、分析、综合、评定; 在情感领域, 学习者根据兴 趣、喜好或任务要求, 主动参与社会学 习活动, 独立思考、积极实践, 日渐形成自己的价值 观念和体系, 并能够使自己的个性化价值观反作用于 社会实践, 概括起来, 就是接受、反应、形成价值观 念、组织价值观念、价值观念个性化; 在动作技能领 域, 通过模仿、尝试错误等, 通过反复练习产生心里 定向, 内化为精准、连贯协调的习惯, 并能够根据具 体的情景，创造性地改善技能。

为了实现预期学习产出, 所有的教学计划和课程 内容都是遵循 “回溯式设计” 原则, 并由此延伸开来, 在教学设计的过程中, 以学习产出为重要质量准则, 将学生表现作为教学成果的评量依据, 实现完整的匹 配矩阵。

\section{3 评价的方法}

伴随着 “成果导向” 认证标准这一转向, 如何构建 与 $\mathrm{OBE}$ 理念相匹配的教育目标评价范式成为关键。基 于 “产出式” 的教学目标评价范式在构建合理的教学 目标达成度所采用的评价方法是多元化量化考核法。

为确定学生的课程学习成果, 课程考核依然是重 要 的衡量手段, 为了综合考量学生对知识点的掌握及 学习效果, 多元量化的考核指标应渗透到培养的整个 过程中, 先明确课程具体培养指标点, 根据分解的指 标点, 优化考试内容, 如, 设置摸底测试、章节测试、 随堂考核、中期考核、实践、期末考试等, 并在教学 中结合教学效果评价及时调整, 灵活实施, 根据考核 结果统计指标点达成度, 然后评估课程目标达成度, 形成阶段性的课程 教学评估报告, 最后由评价的结果 促进教学内容的优化, 反馈调节课程的教学设计, 形 成教学反馈的闭环, 这是践行 OBE 教学目标评价范式 的主要途径。

\section{4. $\mathrm{OBE}$ 评价范式的选择及效用}

杜威说, “教育本身无目的, 只是人才有目的”。 赞成 “教育过程内部的问题”、“具体的目的”，反 对 “教育过程以外的目的”、“一般的目的”，基于 “产出式” 的目标范式所追寻的实质, 正是教育过程 内的目的。

\section{1 评价范式选择依据}

基于 “产出式” 的教学目标评价范式作为一种先 进的教育理念, 目前已被世界各地的高等教育尤其是 工程教育领域广泛接受, 其能够被优先选择遵循一定 的科学依据。

\section{1.1 符合教育目的和培养目标的总体设计}

教育目的反映一个国家或地区、各级各类院校培 养人才的质量规格和素质标准, 教学评价, 这就要求 评价范式与教学目标在 “为谁培养”、“培养什么样 的人” 以及 “怎样培养” 等 “质” 的规定性方面存在 一致性, 评价范式的设计和选择不仅满足教育目的需 要, 更要满足国家和社会的总体期望。教育目的的根 本价值在于，促使人潜能的发挥和社会价值的实现， 且社会价值应高于个人价值, 个人只有在有助于社会 发展时才有价值, 即教育的价值应当以其对社会发展 的作用来衡量。评价教育教学需综合考虑人与社会的 关系, 不仅要满足个人的发展需求, 更要注重发展教 育的社会效益。

基于 “产出式” 的目标评价范式强调对人才培养 的质量和规格要求明确, 倡导研究课程运行的整个过 程, 采取全方位的观察方法, 注重课程目标这本身的 评价, 较好地弥补了结果评价的不足, 重视教学环境 的普遍性和非典型性, 不仅解答了 “是什么”, 更要 解答了 “为什么”。

\section{1.2 符合人类认知和教育学心理学规律}

习近平主席在十九大报告中提出, “优先发展教 育事业”, “实现高等教育内涵式发展”。所以, “发展更高质量更加公平的教育”, “全面提高质量” 是教育工作的战略主题。[3] 素质教育的实践要义在于 形成人的健全个性, 其精髓是人的全面发展教育, 即 “五育”（德、智、体、美、劳）不可偏废, OBE 目 标评价认证规范追求 与其实质相当的内涵，要求课程 体系中的每门课程都要对实现能力结构有确定的贡献。 人身心发展具有顺序性、阶段性、不均衡性和差异性 等特点, $\mathrm{OBE}$ 的教学目标评价范式聚焦在学习成果上, 根据每个学生通过努力能够能达到的程度标准, 赋予 从低到高的不同评定等级，进行有针对性的目标达成 度评价, 通过对学生学习状态的明确掌握, 为学校和 教师改进教学提供参考, 且能够将评价结果及时有效 反馈, 根据学生的学习进程进一步将不同层次的学生 划分不同的学习阶段, 并确定出每个阶段的学习目标, 这些具体的、可操作的学习目标也是遵循从初级到高 级、从低水平向高水平的发展规律, 最终目的是为获 得顶峰性的教学成果。

\subsection{3 师生“双主体”的共同诉求}

教学涉及教师和学生两个主体, 教师教学质量很 大 程度取决于教师自身的要求和内在积极性; 学生学 习质量取决于学生学习的积极性和主动性, 与传统 “满堂灌”、“填鸭式”的课程体系与教学模式不同, OBE 强调师生 “双主体” 的地位和作用, 在这一理念 中, 一切教学活动需综合考虑市场需求的人才导向, 
根据实际情况及时调整培养方案、优化资源配置, 动 态设计教学目标, 围绕学 生的知识、能力、素质需达 到的指标全面构建新的课程内容, 并建立教学质量监 控与评价的标准体系。

人的思维需要理性的收玫, 人类的一切可序列化 的或具有序列化倾向的思维最终都要聚焦于理性思维。 课程教学大纲的 “课程目标与教学内容、方法的对应 关系” 部分，应该明确指出教学内容与其所支撑课程 目标的对应关系, 以便于后续规划、统计各课程目标 所占的分值。就人的情感、态度和价值观等方面而言, 教学目标评价 需由理性的规范满足师生 “双主体” 的 共同诉求, 重视学生身心发展的理性, 或延拓教师自 我经验积累的理性, 或者是把几者进行重组, 深入分 析师生的教学行为和结 果, 构建多维综合评价指标体 系, 形成对学生学习质量和老师教学质量的精准评价 方法, 有针对性地为教师和教学管理部门提供科学优 化的教学决策指导, 完善质量反馈, 从而聚合课堂评 价理性, 规范课堂评价的理论研究或实践活动。

\section{2 评价的功能}

评价作为新一轮教学改革的重点, 明确指出改变 课程评价过分强调甄别和选拔的功能, 发挥评价促进 学生发展、教师提高和改进教学实践的功能。我们期 望通过教育获取使人幸福的知识与能力, 不仅能适应 眼前社会生活，同时也能为未来生活做准备.

\section{2.1 反馈-调节功能}

教学评价对教学过程有监督和控制作用，全面、 科学的评价工作通过对学生课堂表现、课业成绩进行 成因分析, 课业有效掌握教学各方面的情况, 评估教 师在多大程度上实现了教育目的层面的法定知识传授, 学生在多大程度上实现了“师定课程” 的教学目标, 从而判断它的成效和缺陷、矛盾和问题。如, 通过分 析学生课业成绩不良的原因, 判断其影响因素, 可能 涉及到家庭、学校、社会, 具体到个人而言, 可能涉 及到智力因素或非智力因素, 学习动机或学习策略、 方法等的影响, 抑或是两者兼而有之。

教学评价如同体格检查, 基于“产出式”的目标评 价范式是对教学观状进行--次严谨的科学诊断, 除了重 视成因分析, 还重视定义认证品质。以便为教学的决 策或改进指明方向。比如, 加拿大工程认证委员会认 证哲学在传统上是基于输入的, 集中于课程内容、班 级规模、资源投入等内容。OBE 教学模式关注的重点 是学生的学习成果而不是教授内容, 强调以学生为主 体, 实现教学范式由“掌握知识”向“具备能力”的根本 转变, 它式的认证标准也是基于学生产出的, 评价主 体多元化、方式多样化、内容全面化、实际全程化, 即 Plan（清晰 地描述学生产出的能力）、Do（实现学 生能力) 、Check (持续地评估学生学习产出) 和 Act
(改善教学的能力), 课程设计者应根据教学的实际 需要, 从整个设计的任何一个步骤起步, 向前或向后, 体现灵活、实用性，并清晰地制定出毕业生的知识、 能力和职业素养等。

\subsection{2 激励一动机功能}

评价对教学过程有监督和控制作用, 对教师和学 生则是一种促进和强化, 使人产生内在的驱动力以朝 着所期望的目标前进。通过评价反映出教师的教学效 果和学生的学习成绩。经验和研究都表明, 在--定限 度内, 经常进行记录成绩的测验对学生的学习动机具 有很大的激发作用、这是因为, 较高的评价能给教师、 学生以心理上的满足和精神上的鼓舞, 可激发他们向 更高目标努力的积极性; 即使评价较低, 也能催人深 思, 激起师生奋进的情绪, 起到推动和督促作用。

OBE 强调绩效责任, 按照岗位群的需要, 层层分 解以确定从事行业所应具备的能力, 然后再由学校组 织相关教学人员, 以这些能力为目标, 设置课程、组 织教学内容, 最后考核是否达到这些能力要求。尽管 OBE 目标评价范式为学生设置标准参照学习的目标, 但并没有提出对特定的教学方式提出需求, 而只对学 生最后学习产出提出要求, 认为只要能够实现学习的 预期产出, 学生可以在不同教育机构中获得学习经验, 可以参与不同类型的教育经验活动, 采用不同的学习 方式、方法。在这一理念的引领下, 学生可以清晰地 感受到来自教师的期望, 感受到通过自己持续的努力 达成的目标而获得获得成就感的体验, 可以清晰的感 受到自己付出的收获。通过持续的教学评价, 可使教 学活动的过程朝着特定的教学目标迈进, 有利于建立 开放、透明、富有弹性和互认的教育结构学生，构建 积极的课程评价体系, 充分发挥评价的激励功能, 对 于促进学生生动活泼、健康地成长有着重要的现实意 义。

\subsection{3 诊断教学问题, 改善教学效果}

评价的结果必然是一种反馈信息，这种信息可以 使 教师及时知道自己的教学情况, 也可以使学生得到 学习成功和失败的体验, 从而为师生调整教与学的行 为提供 客观依据。教师据此修订教学计划、改进教学 方法、完善教学指导; 学生据此变更学习策略、改进 学习方法、增强学习的自觉性。教学评价有利于使教 学过程成为一个随时得到反馈调节的可控系统, 使教 学效果越来越接 近预期的目标。

结合国际工程教育改革及工程教育认证发展趋势, 我们也对 $\mathrm{OBE}$ 教育目标评价范式进行了一些了实践、 探索与改进，制定了一系列专业层面的预期 “学习产 出”, 对课程目标达成度进行定量和定性的分析与评 定; 通过一体化课程设计, 建立与培养目标及课程标 准相匹配的矩阵; 确定课程层面的预期 “学习产出”, 
并设计相适 应的教学策略; 做好专业层面与课程层面 的实际 “学习产出” 评估, 如, 在 OBE 课程教学大纲 的 “学生成绩评定方法” 中, 指明平时成绩、课终成 绩、小测验等项目的所占分比, 明确各项考核应达到 的具体目标分值, 并详尽说明各项考核内容所支撑的 课程占比及权重。

通过基于 “产出式” 的目标评价范式, 教育管理 者可以及时了解学生是否达到或实现阶段性教学目标, 根据结果及时调整资源配置、师资培训和学生辅导等 工作。同时, 通过向家长、教育行政部门和社会公众 等利益相关者展示学生发展情况, 提供努力改善教学 质量的证据, 可以增进高等教育质量 “透明性” , 有 利于实现高等教育问责。

\section{项目基金}

本文为湖南省教育科学 “十三五” 规划 课题《发展 性院校评价的策略与方法研究》 (XJK18BGD001) 的阶段性 成果之一。

\section{REFERENCES}

[1] Yin, X.Y, Wang, X.J. (2019) Research on the evaluation method of objective achievement degree of operating system principle course under OBE concept. J. Computer age, 9: 84-85.

[2] Cheng, S.X. (2004) Educational evaluation methods and techniques. Beijing normal university press. BeiJing.

[3] (2016) To comprehensively improve the national education quality and accelerate the modernization of education with the new development concept -- speech by minister yuan guiren at the 2016 national education work conference.J. The people's education, (Z1):8-21. 